\title{
Selected-Area Small-Angle Electron Diffraction
}

\author{
G. S. Y, YEH*, P. H. GEIL \\ Department of Polymer Science and Engineering, Case Institute of Technology, Cleveland, \\ Ohio, USA
}

Received 15 May 1967

\begin{abstract}
Selected-area electron diffraction capable of resolving spacings up to $2000 \AA$ from first-order discrete reflections has been achieved using a standard, double-condenser electron microscope. The technique allows photographing of the selected area, at sufficient magnification, that gives rise to the small-angle scattering pattern, in addition to the normal capabilities of obtaining related wide-angle diffraction and wide-angle and small-angle dark-field micrographs. Most, but not all, of the results of discrete and diffuse, small-angle electron diffraction studies from a large variety of specimens including drawn, annealed polyethylene, latex particles, evaporated gold particles, grating replicas, and slit edges have been explained on the basis of the structures observed in the corresponding electron micrographs. Small-angle electron diffraction is found to be more sensitive to defects in the packing of the scattering centres than small-angle $X$-ray scattering.
\end{abstract}

\section{Introduction}

In recent years, there has been a great deal of research on the fine structure of polymers using small-angle X-ray diffraction. The problems have recently been reviewed by Geil [1] and by Statton [2]. Besides the stringent experimental requirements involved in obtaining small-angle X-ray diffraction patterns, there also exists the problem of the interpretation of the patterns in terms of the morphology of the sample. The only exception is the polymer single-crystal mat; the pattern obtained from single-crystal mats, interpreted as Bragg diffraction, yields a spacing which is in excellent agreement with observed, electron microscope, step-height measurements in both as-crystallised samples and annealed samples. In the case of bulk samples, the small-angle spacings are not orders of each other. The lack of an exact ratio of 2:1 may be due to a distribution of sizes [3], but there is no observed structure with a periodicity or size equal to the largest spacing [1]. There are also problems in the interpretation of patterns from oriented polymers, in particular the origin of both the line and four- point patterns. The technique discussed here was developed during the course of our study of oriented polyethylene terephthalate (PETP) [4], in which it became necessary to interpret such patterns.

In all small-angle diffraction studies, it would be particularly useful if one could obtain a small-angle diffraction pattern simultaneously with viewing, at sufficient magnification, the corresponding area giving rise to the scattering. Such a possibility is inherent in an electron microscope. The instrument has a highly monochromatic beam which can be made perfectly parallel. The requirement of large camera lengths is easily fulfilled through corresponding enlargement of the diffraction image. Such a possibility was first explored by $\mathrm{Mahl}$ and Weitsch [5] and later by Bassett and Keller [6]. The principles behind their methods were similar: in Mahl and Weitsch's case, the objective lens was used for collimating the beam, and the specimen was placed in the high-resolution diffraction position; Bassett and Keller simply used the double-condenser system for collimation of the beam and did not energise the urgical Engineering, University of Michigan, Ann Arbor, *Current address: Depart
Michigan 48104, U S A 
objective lens. The specimen was in the normal position. The essential difference in the two techniques is in the maximum, obtainable, effective camera length, which is closely related to the achievable resolutions in the diffraction pattern. Bassett and Keller's method permits a much higher resolution as a result of the longer camera length. Both methods allow some shadow-cast-type imaging of the specimen at a very low magnification $(\times 500)$. Although, in Bassett and Keller's technique, high-resolution microscopy is readily feasible by energising the objective lens, correlation of the examined area and the area giving rise to the scattering pattern relies on an additional low-magnification photograph serving as a marker.

The present technique is based on the simple relation: $L=f_{0} \cdot M_{\mathrm{i}} \cdot M_{\mathrm{p}}$, where $L$ is the effective camera length, $f_{0}$ is the objective focal length, and $M_{\mathrm{i}}$ and $M_{\mathrm{p}}$ are the magnifications of the intermediate and projection lenses. The objective lens is used in a normal fashion. The required, much greater camera length is achieved by increasing the objective focal length by raising the specimen to a higher position and reducing the objective current. The advantage of this technique, which is essentially the difference between the present technique and earlier ones, lies in the possibility of photographing the selected area giving rise to the small-angle scattering at reasonably high magnification and resolution and, in addition, being able to obtain related wide-angle diffraction and wide-angle and small-angle dark-field micrographs.

The immediate objective of this work was to understand the diffraction patterns from oriented PETP [4] by examining oriented structures, some with clearly defined morphology and some with morphology which is still undergoing intensive study. The results of that study are to be published shortly. In this paper, we describe the technique and its initial application to the small-angle diffraction from a wide range of subjects in order to show its versatility.

\section{Technique}

The technique involves no basic modifications in the electron microscope. The only requirement, besides the availability of a doublecondenser electron microscope capable of doing selected-area diffraction, is that there must be room enough to place the specimen a few centimetres above the normal specimen position. The larger distance is needed to permit one to increase the focal length of the objective lens and, therefore, the effective camera length. In the Hitachi HU-11A microscope used in all the work reported here, this requirement is easily satisfied.

Fig. 1 is a selected-area small-angle scattering ray diagram. It is the same ray diagram used for normal wide-angle selected-area diffraction, except for the raised specimen position. To raise the specimen to a specified position, a slightly modified specimen holder was made. A picture of our holder is shown in fig. 2 . On the right is the cold-stage specimen holder for normal specimen position and on the left the modified specimen holder with the collar insertion ring removed to expose the modified portion. A cold-stage specimen holder was modified because the cold stage was in continuous use at the time. The slightly modified specimen holder can be used either for highresolution electron microscopy work, by placing the specimen at position 1 , or for small-angle work, by placing the specimen at position 2 .

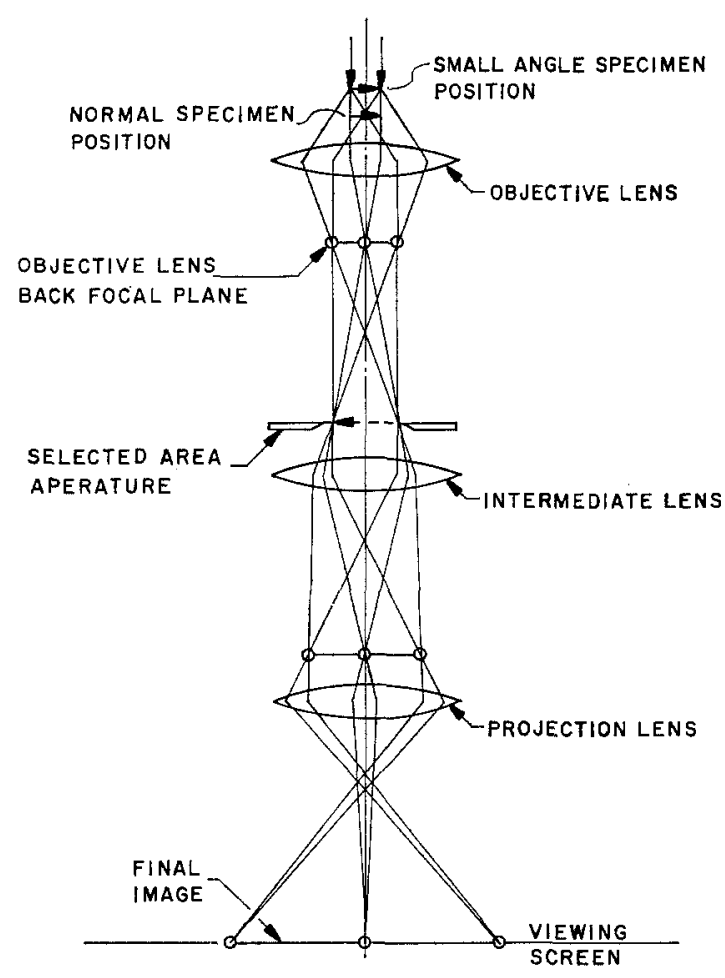

Figure 1 Schematic drawing of the selected-area smallangle electron diffraction ray diagram. 


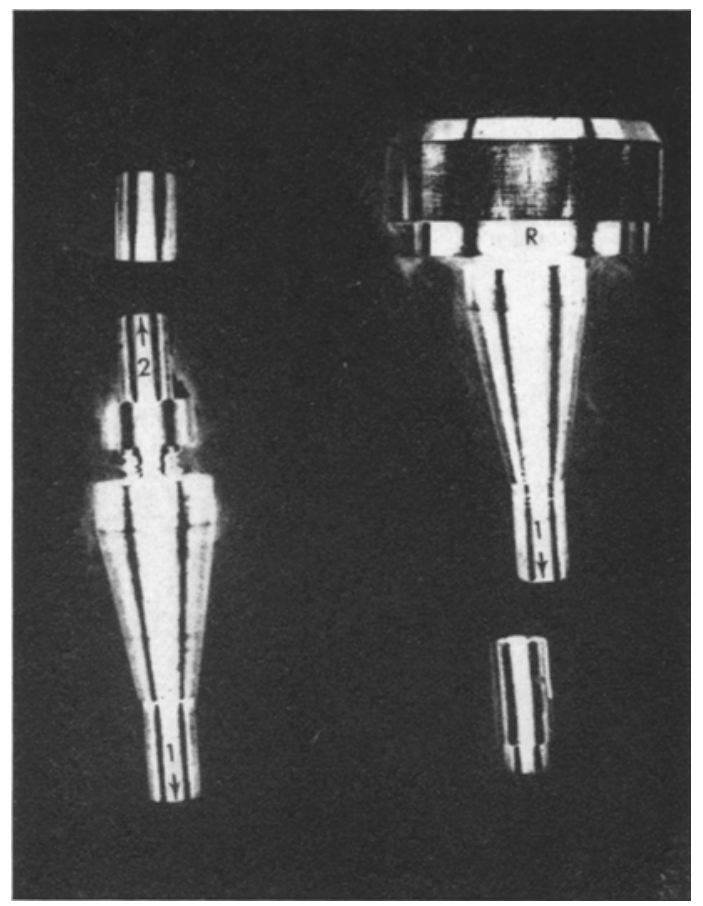

Figure 2 Picture of the specimen holder (left) used for small-angle diffraction work with the collar insertion ring, $R$, removed for clarity. The small-angle specimen position, 2 , is about $3 \mathrm{~cm}$ above the normal specimen position, 1. On the right is the normal specimen holder.

The change in specimen position results in a change in the objective current required for focusing. It will be lower for the same objective pole-piece than in normal operation. For example, at an accelerating voltage of $75 \mathrm{kV}$, the objective focusing current is about $71 \mathrm{~mA}$. By raising the specimen approximately $3 \mathrm{~cm}$ above the normal specimen position, the focusing current changes to about $36 \mathrm{~mA}$ (a weaker objective lens with a large bore size $(24 \mathrm{~mm})$ has also been used, permitting the use of higher focusing currents). Another consequence of this change in specimen position is a loss in resolution in the image $(\sim 100 \AA$ at the small-angle position) owing to the unfavourable position of the specimen. Various specimen positions, including as high as $6 \mathrm{~cm}$ above the normal, have been tested. A position about $3 \mathrm{~cm}$ above the normal specimen position corresponding to an effective camera length of about $12.5 \mathrm{~m}$ was found to be convenient for the work reported here. Exact camera lengths have to be internally calibrated each time using suitable standards placed directly on the same specimen grid. One such standard is the $9.3 \AA$ reflection of poly-4-methyl-pentene-1 (P4MP1) single crystals, previously used by Bassett and Keller [6] in their small-angle work. Other standards, such as gold colloidal particles deposited by means of vacuum evaporation, which have a diffuse maximum corresponding to a Bragg spacing of about $100 \AA$, were found to be useful in samples that are susceptible to attack by solvents. The gold particles have to be calibrated independently. Fig. 3 shows both the small-angle diffraction pattern of gold and the $9.3 \AA$ reflection of P4MP1. It was taken using a smallerthan-normal projection-lens current to include both patterns on the same negative.

In addition to the required, much larger effective camera length for high-resolution small-angle scattering work, the primary beam at the film position has to be limited to very small sizes. This was achieved by reducing the selected area of the sample to a minimum and by keeping the selected-area aperture clean. A small and very clean objective aperture in the usual selected-area blades position was used. A

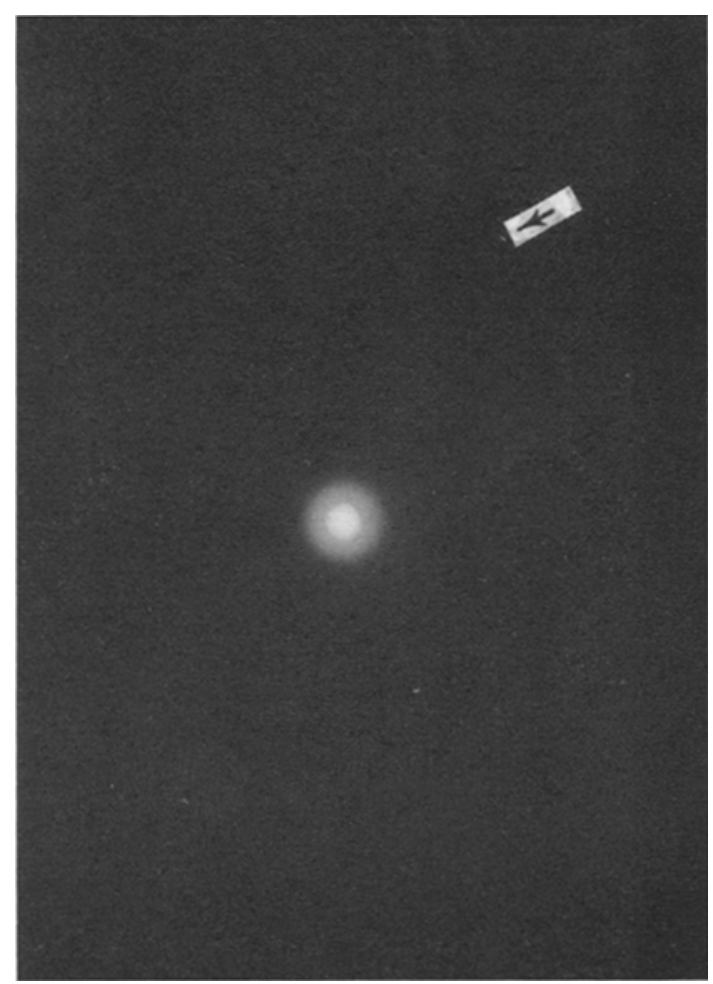

Figure 3 Small-angle electron diffraction patterns of gold and the $9.3 \AA$ spot reflection of P4MP1 (arrow). 
smaller condenser aperture will also help to cut down the central beam spot, but this is normally not required unless the specimens are highly susceptible to damage by the beam.

The procedure employed for doing smallangle diffraction was the same as that for normal wide-angle diffraction. After placing the specimen at the small-angle position, an area was selected, focused, and photographed, the microscope was then switched to diffraction position and the corresponding small-angle scattering pattern of the previously selected area was recorded. Dark-field studies were made in the same fashion as ordinary dark-field microscopy: that is by placing an objective aperture over a selected portion of the small-angle scattering pattern and switching back to the microscopy position. To record wide-angle diffraction patterns, the magnification had to be reduced either by decreasing the projection lens current or by changing the projection polepiece to a weaker lens, or by doing both.

Bragg spacings as large as $2000 \AA$ have been achieved routinely without much trouble. In our opinion, the ultimate resolution could be at least twice that obtained here, judging from the central beam, which can be limited to very small sizes by the selected-area aperture.

\section{Applications and Results}

\subsection{Oriented Polymers - Striated Structures}

As mentioned earlier, it was during the course of the work on the morphology of straininduced crystallisation of polyethylene terephthalate that the present technique was developed. Results on oriented PETP [4] and on highly drawn, linear polyethylene [4] using this technique are to be reported shortly. The technique was first applied to oriented specimens of known morphological features, such as the striated structures [7] which develop during the annealing of fibres pulled from polyethylene single crystals. It has been reported by Fischer and Schmidt [8], using small-angle X-ray studies, that the long-period spacing of polyethylene fibres increases with annealing temperature and annealing time. The striated structures, which presumably give rise to the long period, have been shown by Geil [7] to be essentially chain-folded single crystals of up to $1000 \AA$ in lateral size and to have thicknesses of the order of $250 \AA$ that depend on the annealing temperature. At present, there are still unsolved problems regarding the formation of these striations and their relationship to the original structure of the fibre before heat-treatment. These will be discussed only in relation to the application of the present technique and the interpretation of the scattering phenomena. Further work will be needed in order to solve the problem of their formation.

Preparation of the striated structures was done by first shearing polyethylene single crystals between two glass slides to produce the fibres. Polyethylene single crystals were used because they have been well characterised [9]. The fibres produced on shearing were then annealed in air in a temperature-controlled glass tube, removed, and prepared for electron microscope studies.

The fibres before annealing had a barely visible periodicity of about $140 \AA$ along the fibre axis. The $140 \AA$ periodicity corresponds closely to the fold period of the crystals used. Similar observations of striations in freshly drawn fibres have been previously reported by Fischer and Schmidt [8]. The fibres gave a welloriented, wide-angle electron diffraction fibre pattern but no small-angle diffraction in the electron microscope.

Following annealing, well-defined striated structures were observed. An example is given in fig. 4, showing the bright-field, the darkfield, and the small-angle scattering pattern of the selected area. Both electron micrographs were taken at the small-angle specimen position at a magnification of about $\times 6600$ using a $24 \mathrm{~mm}$ bore-size objective pole-piece. The resolution as seen from these electron micrographs is reasonably good and useful in terms of the size of the structure normally encountered in small-angle work. The line-type diffraction maxima observed on the meridian correspond to a periodicity of $385 \AA$, which agrees with the centre-to-centre distance of about $378 \AA$ of the striations well within the experimental error of $\pm 5 \%$. Shadowing with metal does not change the periodicity, but it does increase the intensity of the maxima. A second-order maxima was observed in the original negative. Higher orders of reflections, up to as many as three, have been observed in some preparations. They are orders of each other (i.e. first, second, and third), and their intensities decrease in the expected manner also. It is interesting to note from the dark-field electron micrograph that the striated structures form a paracrystalline lattice [10] with only a few repeats per lattice. No 


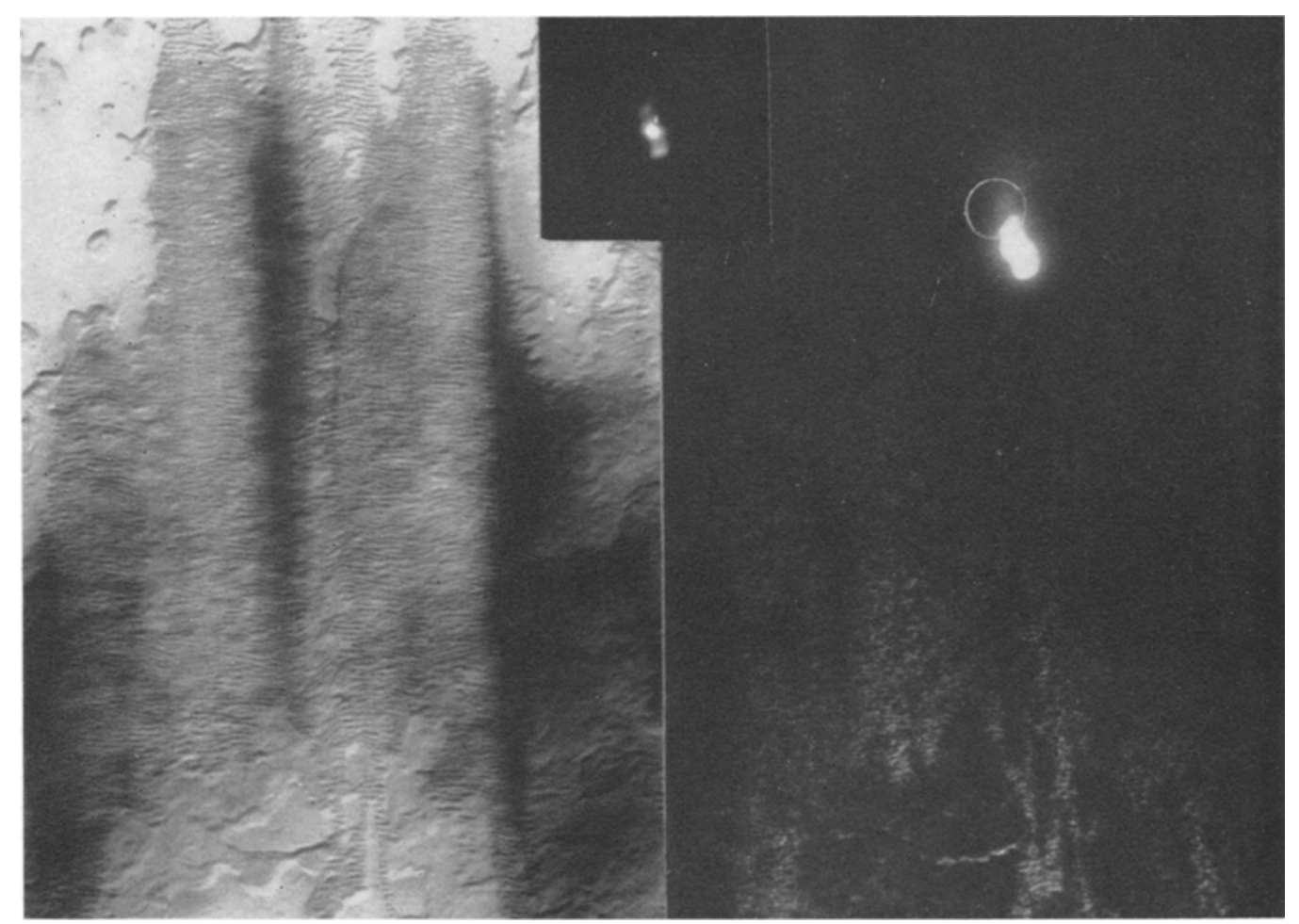

Figure 4 Selected-area electron micrographs of annealed, drawn polyethylene taken with the sample at the small-angle specimen position showing the $378 \AA$ striated structures under both bright-field and dark-field illumination. A smallangle electron diffraction pattern from the selected area is also given. The dark-field micrograph was obtained by placing an objective aperture over one of the discrete reflections, as indicated by the circle.

equatorial diffuse scattering was observed, as expected from their corresponding micrographs.

The specimen shown in fig. 4 was obtained by annealing the fibres at $128^{\circ} \mathrm{C}$ for about $20 \mathrm{~h}$. Several other annealing temperatures and times were also used to study their effects on the width of the striations. The results (table I) showed that there is an increase of long period with annealing temperature and annealing time, and that the long period measured from the smallangle pattern agrees within $\pm 5 \%$ with the centreto-centre distance in the corresponding electron micrograph. The electron microscopy measure- ments of the centre-to-centre distance were obtained by taking a mean of a hundred measurements. No electron microscope measurements were made of the samples annealed at $130^{\circ} \mathrm{C}$. The small-angle electron diffraction long periods of these striations are in reasonable agreement with small-angle X-ray diffraction measurements on drawn polyethylene samples annealed for similar times and temperatures $[8,11]$.

It is interesting to note that the striated structures began to form at about $110^{\circ} \mathrm{C}$ after $24 \mathrm{~h}$ (fig. 5a), but that these gave no small-

TABLE I Small-angle electron diffraction long period of striated structures.

\begin{tabular}{lrll}
\hline Temperature $\left({ }^{\circ} \mathrm{C}\right)$ & Time $(\mathrm{h})$ & Long period $(\AA \pm 5 \%)$ & $\begin{array}{l}\text { From electron } \\
\text { microscope }(\AA)\end{array}$ \\
\hline 123 & 18 & 330 & 337 \\
128 & 5 & 365 & 350 \\
128 & 24 & 430 & 450 \\
131 & 5 & 412 & \\
131 & 17 & 495 & \\
\hline
\end{tabular}




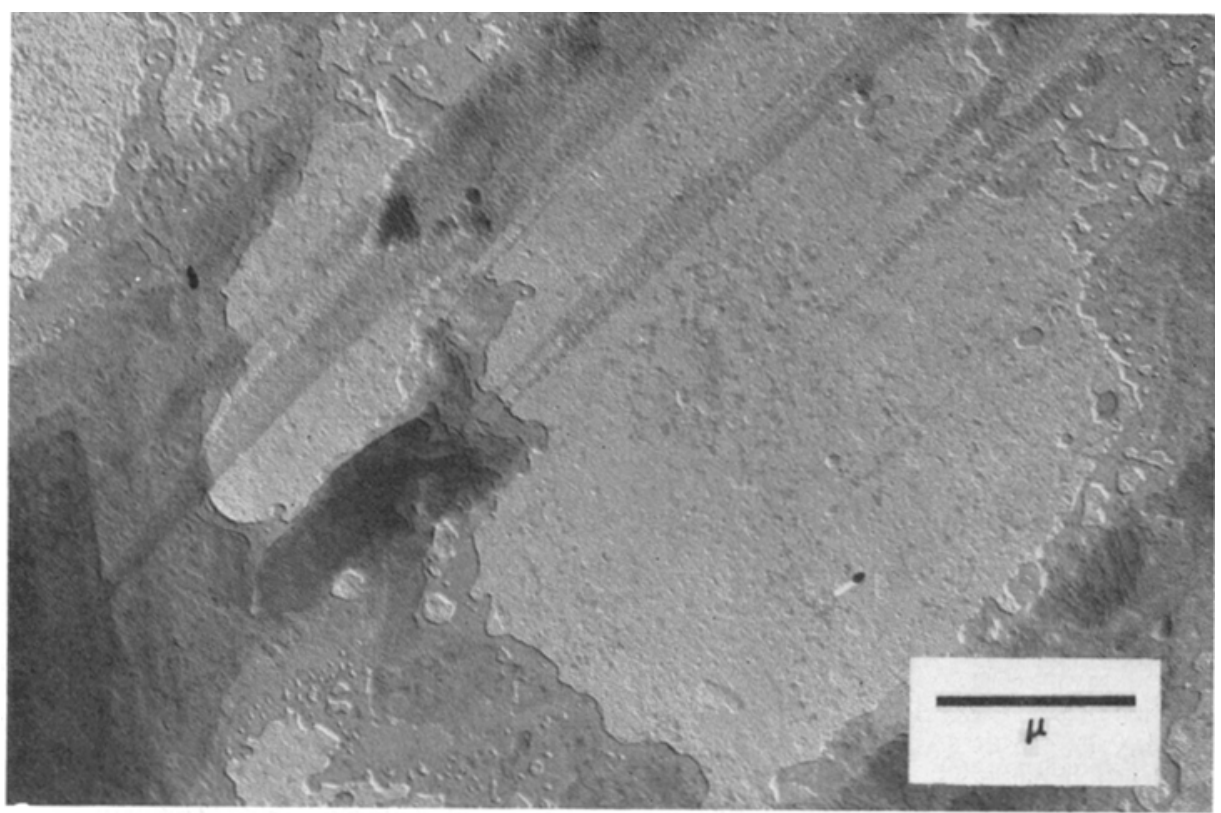

(a)

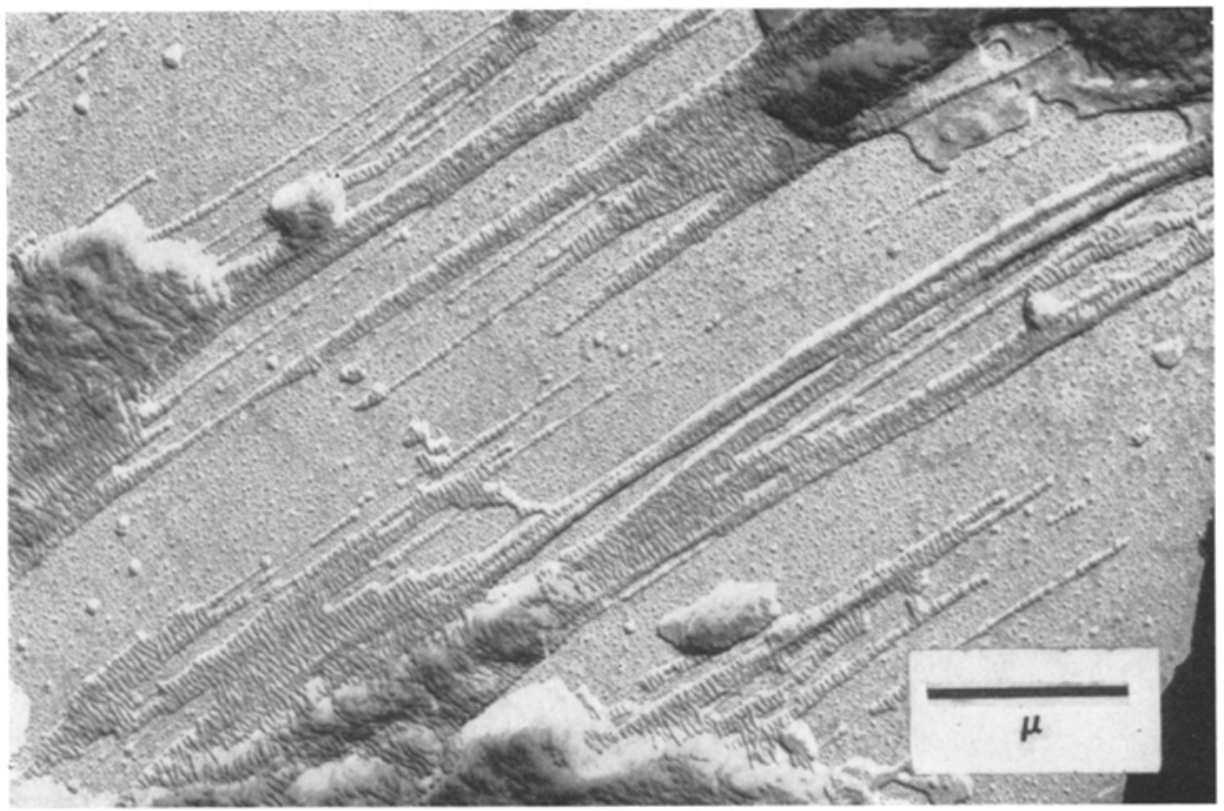

(b)

Figure 5 Electron micrographs showing the striated structures formed at two different annealing temperatures: (a) $110^{\circ} \mathrm{C}$ for $24 \mathrm{~h}$; (b) $118^{\circ} \mathrm{C}$ for $21 \mathrm{~h}$. 


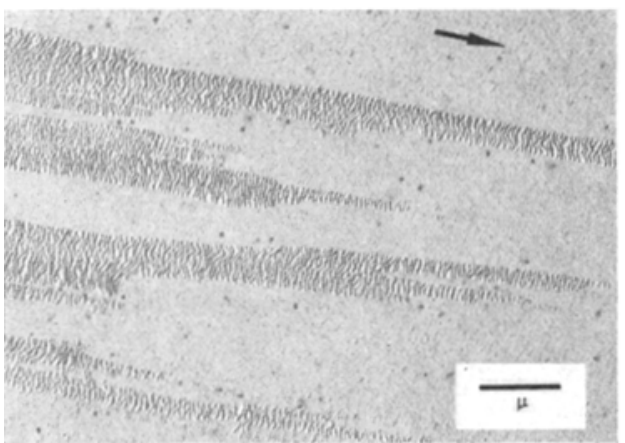

(a)
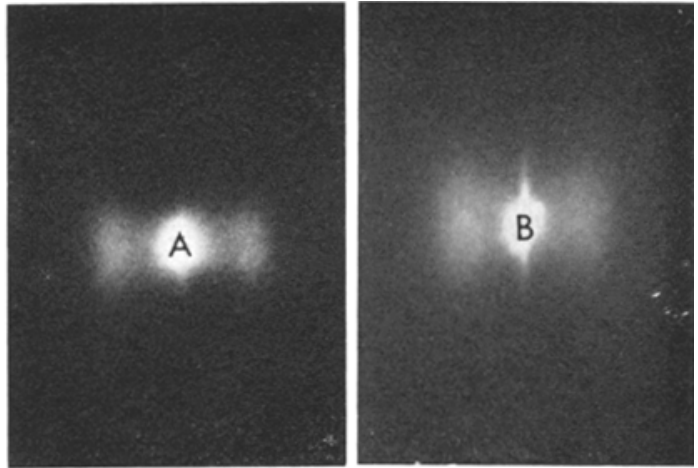

(b)

Figure 6 (a) Striated structures on Mylar which was drawn 20\%; average spacing between them is $560 \AA$. (b) Smallangle electron diffraction patterns from similarly striated structures: A, $20 \%$, small-angle spacing is $500 \AA$; $B, 40 \%$, small-angle spacing is $630 \AA$.

angle electron diffraction patterns. The same holds true for samples annealed at $118^{\circ} \mathrm{C}$ for $21 \mathrm{~h}$ (fig. 5b). It was not possible to obtain small-angle electron scattering patterns from these structures, although seemingly very welldeveloped striations $(\sim 250 \AA)$ are observed in the electron micrograph. An X-ray long-period pattern would be expected from similar fibres annealed under similar conditions [8]. Possible causes are discussed below.

Finally some data were obtained on these structures after they had been stretched on Mylar. The effect of stretching on long period is of particular interest since the thickness of the lamellae, which changes on annealing, is now kept constant whereas the spacing between them should change. Specimens were prepared in the same fashion except the crystals were deposited on a 1 mil Mylar film, sheared with a glass slide, and annealed at $123^{\circ} \mathrm{C}$ for $\frac{1}{2} \mathrm{~h}$ to give an original periodicity of about $260 \AA$. After annealing, the film was stretched along the fibre direction to a given extension. The striations remain almost perpendicular to the substrate and are clearly seen to draw some distance apart (fig. 6a). These were obtained by drawing the Mylar substrate to an overall extension of $20 \%$. However, the observed centre-to-centre distance increased by almost $100 \%$ as did the corresponding small-angle periodicity. For a given extension of the Mylar substrate, several different small-angle periodicities were obtained on the same specimen, suggesting considerable non-uniformity in the local drawing characteristics of the annealed substrate. This nonuniformity is not observed using unannealed substrates. The higher the draw ratio, the greater is the separation of the striated structures, resulting in a larger small-angle spacing (table II). Examples of small-angle patterns from samples drawn on Mylar by 20 and $40 \%$ are shown in part $A$ and part $B$ of fig. $6 \mathrm{~b}$ respectively. The slight, equatorial diffuse scattering in the pattern of $\mathbf{B}$ is probably due to some fibres still present after annealing.

TABLE II Small-angle electron diffraction long period of striated structures on Mylar substrate drawn to various extensions.

\begin{tabular}{cl}
\hline $\begin{array}{l}\text { Substrate } \\
\text { extension }(\%)\end{array}$ & $\begin{array}{l}\text { Long period } \\
(\AA \pm 5 \%)\end{array}$ \\
\hline 0 & 260 \\
20 & 425,215 (2nd order) \\
20 & 550,275 (2nd order) \\
20 & 500 \\
40 & 630 \\
40 & 572 \\
\hline
\end{tabular}

\subsection{Latex Lattices}

Yudowitch [12] determined the mean particle size of uniform latex particles by analysing the small-angle X-ray pattern obtained from suspensions of the particles and showed that the experimental curve was predictable from theory after correction for packing. No such reported studies have been made using electron scattering, although Kiho [13] has privately communicated to us that he has obtained some electron smallangle scattering patterns of latex particles in an electron microscope using a technique similar to Bassett and Keller's. Recently, Woods and Dodge [14] polymerised highly uniform latex particles varying in sizes between about 170 and $12400 \AA$. Some of these particles have a 
G. S. Y. YEH, P. H. GEIL
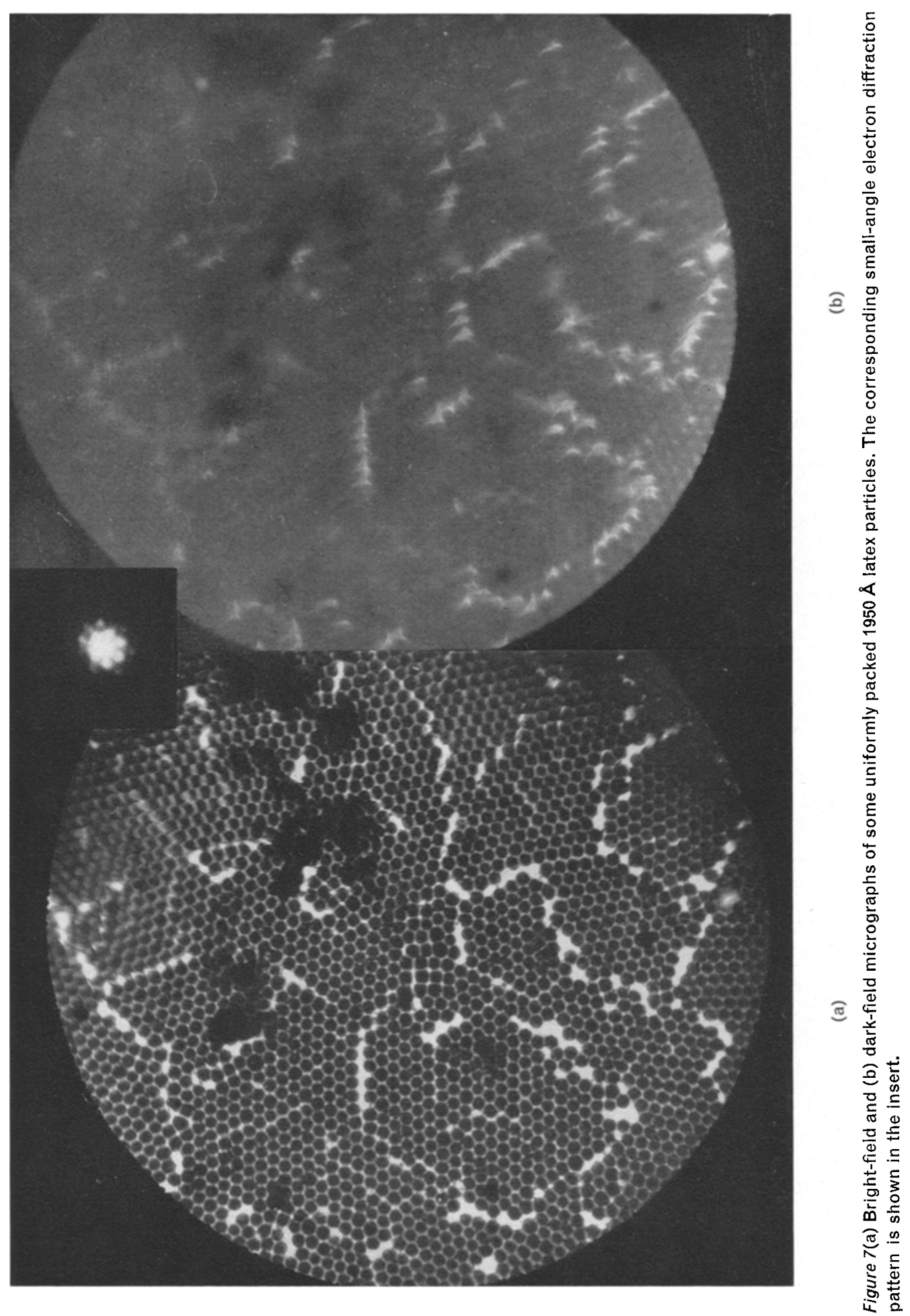
TABLE III Calculated and measured values of small-angle electron diffraction long period, $d$, of latex particles.

\begin{tabular}{cccl}
\hline Diameter $(\AA)$ & $d(\AA)$ (measured) & $d(\AA)$ (calculated) & $(h k . l)$ \\
\hline $700 \pm 75$ & 595 & 600 & $(10.0)$ \\
$1380 \pm 20$ & 1185 & 1200 & $(10.0)$ \\
& 678 & 690 & $(11.0)$ \\
$1950 \pm 10$ & 1650 & 1670 & $(10.0)$ \\
& 970 & 975 & $(11.0)$ \\
& 825 & 838 & $(20.0)$ \\
& 632 & 632 & $(21.0)$ \\
\hline
\end{tabular}

uniformity index of about $1.01\left(\bar{M}_{\mathrm{w}} / \bar{M}_{\mathrm{n}}=1.01\right)$. Under slow drying conditions, these latex particles can be uniformly packed in a hexagonal crystalline arrangement. Their uniformity and the size range available are particularly suitable for application in the present technique. Both discrete and diffuse scattering were obtained. The number of orders of discrete reflections is a strong function of their packing, which in turn is strongly dependent upon their uniformity index as well as the method of preparation. The larger particles were found to pack more uniformly, thus resulting in higher orders of discrete reflections. These findings confirmed the conclusions reached by Woods and Dodge [14].

No diffuse scattering was observed for particles smaller than $4000 \AA$ probably because it becomes too weak to be resolved. Fig. 7 shows bright- and dark-field micrographs of some wellpacked $1950 \AA$ latex particles with the corres-

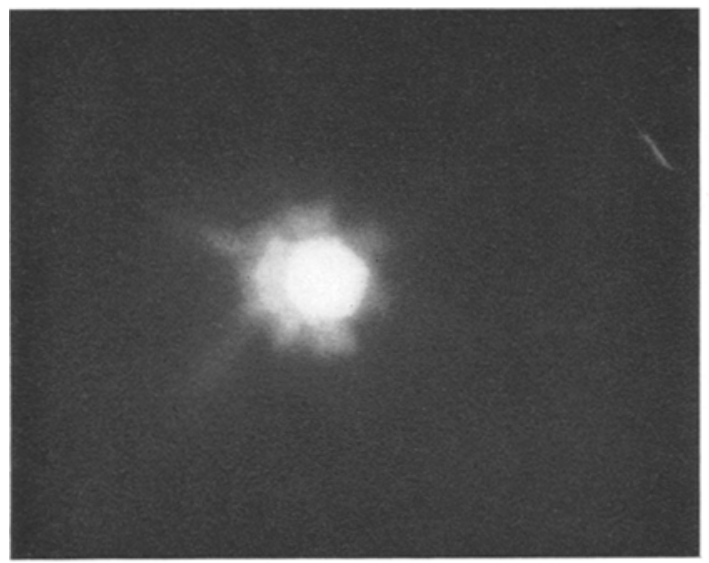

(a) ponding small-angle scattering pattern. This sample was not shadowed. Shadowed samples resulted in some extraneous diffuse scattering due to the shadowing material, the position of the maxima being unchanged. All the reflections could be indexed based on the hexagonal unit-cell of the latex, and the calculated and measured values of $d$ spacings agreed within $1 \%$ of each other (table III). However, one notes from the figure that, although several orientations of the latex lattice are included within the aperture, a single set of reflections is observed. Apparently only a portion of the visible area is contributing to the scattering pattern. In the dark-field micrograph, considerable intensity is seen associated with defects in the packing of the spheres; some of the diffuse scattering in observed diffraction patterns from larger spheres may be related to these defects.

The $4000 \AA$ latex particles give both a discrete and a diffuse type of scattering (fig. 8).

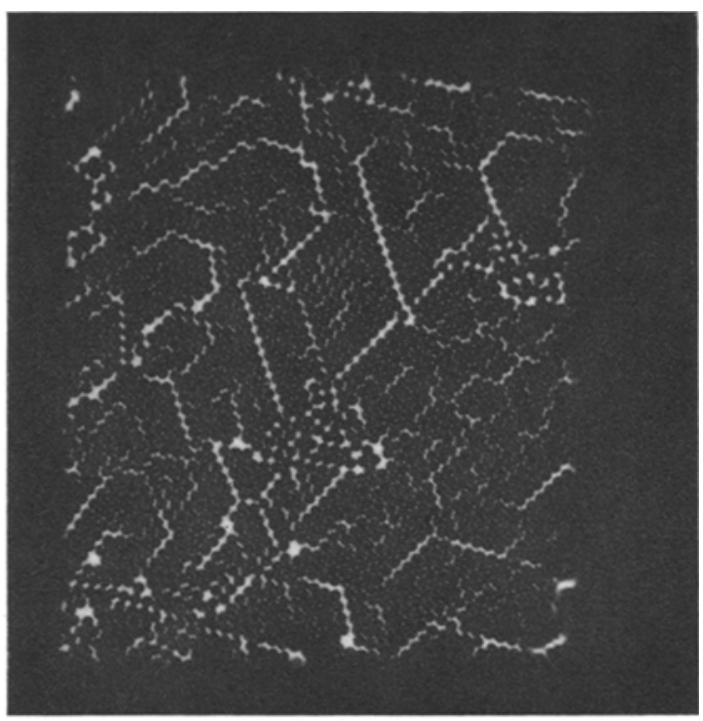

(b)

Figure 8 (a) Diffraction pattern and (b) corresponding bright-field micrograph of $4000 \AA$ latex spheres. 
Comparison with other patterns suggests that the more prominent six-sided diffuse scattering is oriented at the same angles as the first-order discrete reflections which are at too small a value of $\theta$ to resolve. In addition, there are six pairs of much shorter diffuse streaks which lie at an angle of about $20^{\circ}$ to either side of the longer diffuse scattering. The origin of these shorter diffuse streaks is not known at the present time although they may be related to the defect scattering. Dark-field micrographs from these particles show two intense arcs at the edge of each particle bordering a defect. Interesting is the fact that these shorter streaks have been observed only for the $4000 \AA$ size particles. An example of the longer six-sided diffuse scattering is shown in fig. 9 which was obtained from some $12400 \AA$ particles. Dark-field photographs using the diffuse scattering are given in fig. 10 , together with their corresponding selected small-angle scattering patterns. One sees that it is the edges of the particles, where the electron density changes, that are contributing to the diffuse scattering observed.

\subsection{Non-polymeric Samples}

Gold colloidal particles evaporated on carboncoated grids show a fairly sharp diffuse ring,

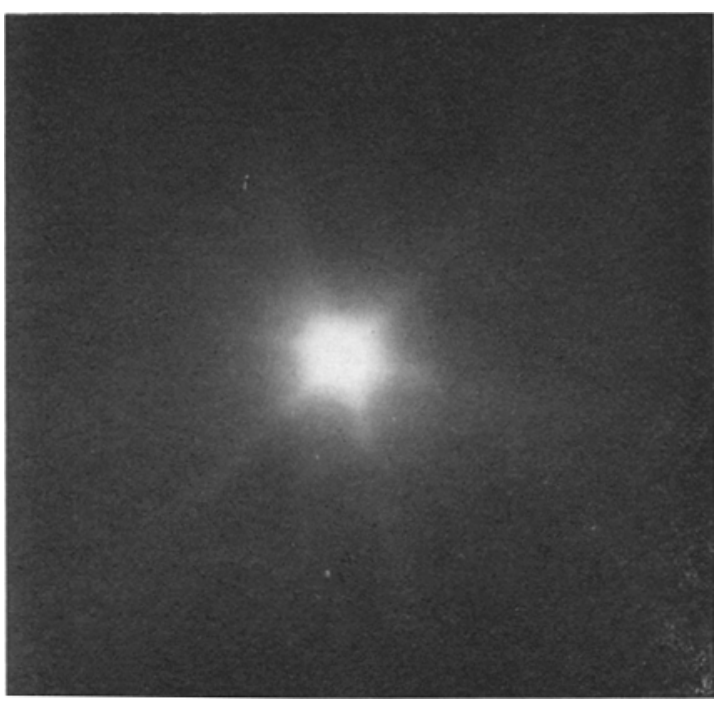

Figure 9 Diffuse scattering from $12400 \AA$ latex particles. The discrete reflections are at too small an angle to resolve.

as shown earlier in fig. 3. The periodicity calculated from the position of the maximum, based on Bragg's equation, is about $100 \AA$ and agrees, within experimental error, with the
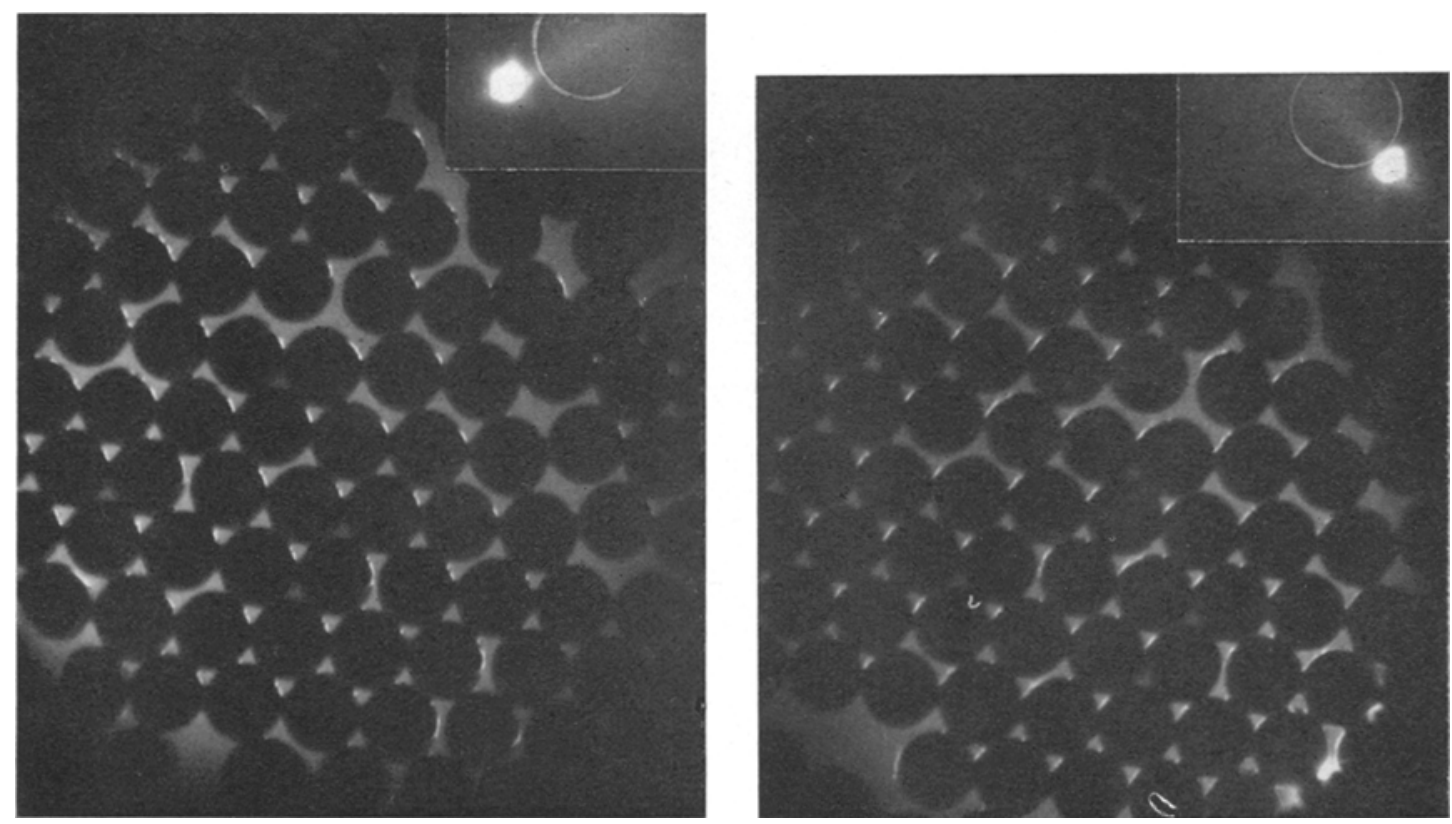

Figure 10 Dark-field micrographs and corresponding diffraction patterns of some $12400 \AA$ latex particles. 


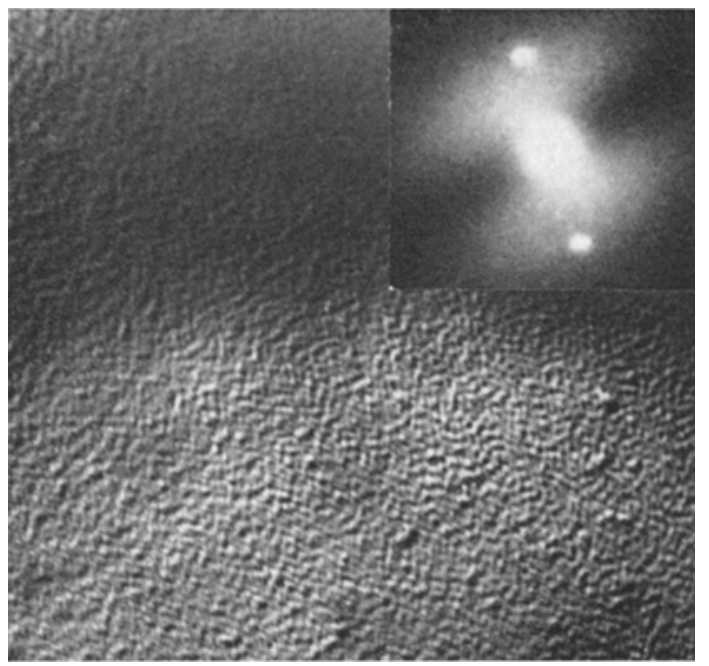

Figure 11 Small-angle line-type electron diffraction pattern and the corresponding replica of the corrugated (110) surface of an aluminium single crystal.

observed particle spacing of $105 \AA$. These are comparable with the results obtained on gold films by Mahl and Weitsch [5].

An irregular-line-type grating replica was obtained from an aluminium single crystal with a corrugated (110) surface, supplied to us through the courtesy of P. E. Doherty of Arthur D. Little and Co*. Doherty and Davis [15] reported the observation of grooves spaced approximately $350 \AA$ apart on the oxidised (110) surface of aluminium single crystals. Fig. 11 shows a small-angle line-type pattern and the corresponding selected area obtained from the replica. The sharp, discrete maximum (a secondorder maximum is present on the original negative) corresponds to a periodicity of $387 \AA$, which again agrees with the repeat distance of about the $375 \AA$ measured directly on the corresponding electron micrograph. The diffuse maximum has slightly larger spacing and, in addition, one notes that the two maxima have a slightly different orientation. The difference in angle corresponds to the oblique shadowing angle. However, we are unable to explain the difference in periodicity or angle unless it is a result of the difference in form factor in the two types of scattering. The grating is seen to be made up of rows of particles, each of which produced an oblong shadow at an angle to the normal to the grating row.

*Address: Acorn Park, Cambridge, Mass, USA
Diffuse scattering from the edges of the collimating slits is the primary limiting factor preventing high resolution in small-angle X-ray diffraction studies. Its source, not completely understood, has been suggested [16] as resulting from a particle-type scattering from the thickness of the edge penetrated by the beam. Slitedge scattering can also be obtained in the electron diffraction patterns. By using an elongated crack in a carbon substrate, the simulated slit gives sharp, diffuse scattering along a direction perpendicular to the crack (fig. 12).
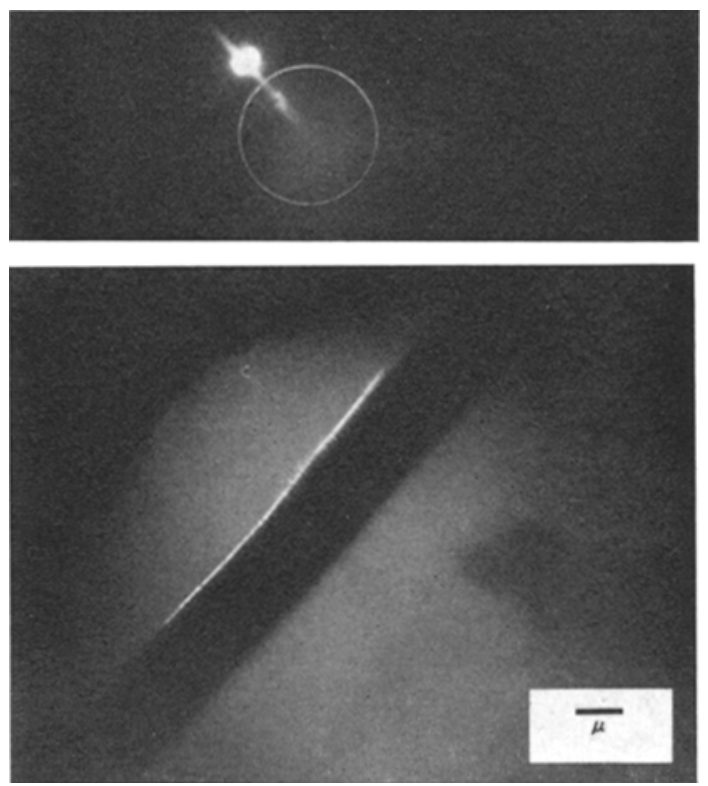

Figure 12 Small-angle electron diffraction pattern and dark-field micrograph of a crack in a carbon film.

The dark-field micrograph taken using the diffuse scattering to the right of the main beam shows that the scattering is coming from the left edge of the slit, the diffuse scattering to the left of the main beam being due to the right edge of the slit. The scattering is seen to arise from the material at the edge and not from the void. The cause of the beaded nature of the darkfield image, particularly obvious at the ends of the edge, is not known. In bright field, the edge is smooth at this magnification.

\section{Discussion}

From the results, it is seen that this technique shows particular promise in the overall study of small-angle scattering phenomena. Most of our 
results are explainable in terms of the structures observed in the corresponding electron micrographs, particularly in those dark-field micrographs showing the origin of scattering. Bragg's equation is directly applicable to discrete maxima and to diffuse maxima (e.g. striated polyethylene fibres, oriented PETP [4], and colloidal gold particles). Higher orders of reflections resulting from the same morphological origin are always orders of one another (i.e. first, second, and third), and their intensities decrease in the expected manner also. Furthermore, the number of repeats along any given direction need not be large for maxima to occur, as judged from the dark-field micrographs, but there should be sufficient density differences between the repeats.

The nature of the diffuse slit-scattering may be related to the anomalous edge-waves in electron microscopy, which were pointed out by Hall [17] in his Fresnel diffraction studies of imaging in the electron microscope. The appearance of these anomalous waves or edge scattering superposed on the normal Fresnel diffraction pattern is predicted from theory by van de Hulst [18]. He showed that the waves specularly reflected off an edge are scattered over a large angle as compared to the Fresnel pattern if the condition $Z>>\left(R^{2} / k\right)^{1 / 3}$ is satisfied (where: $Z$ is the distance between the edge and the screen or the camera length; $R$, the radius of the scattering edge; and $k(=1 / \lambda)$, the reciprocal of the wavelength). This condition is easily satisfied both in the electron diffuse scattering observed from the slit edge (see fig. 12) and in that from the edge of a latex particle (see fig. 10). This may also apply to the slit scattering normally encountered in small-angle X-ray diffraction.

It is of interest to note that polyethylene fibres annealed between 110 and $118^{\circ} \mathrm{C}$ do not give a small-angle electron diffraction long period, even though fairly well-developed striated structures can be formed at these temperatures and an X-ray long-period pattern would be expected from similar fibres annealed under similar conditions. A periodicity was observed to be present even prior to annealing and it has been suggested [19] that it may be due to blocks of folded-chain crystals remaining fairly intact after deformation but having highly distorted fold surfaces which behave as defects in the macrolattice. Sufficient annealing will heal the defects and regularise the highly distorted fold surface. The electrons seem to be more sensitive to defects than X-rays, judging by the degree of annealing required for the appearance of small-angle electron diffraction patterns. Similar observations were made in another study of highly drawn polyethylene [4] using the same technique, where the small-angle electron diffraction long period corresponding to the original lamellae thickness was inexplicably absent. Further studies are needed to determine why small-angle electron diffraction is more sensitive to the presence of defects in the sample than small-angle X-ray diffraction. There are at least two other features of the observed scattering which we are not able to explain satisfactorily at present - the difference in angle and position of the periodicities observed in fig. 11 and the possible relationship of the diffuse scattering in fig. 9 and defects in the latex lattice. Despite these problems, the technique promises to be highly useful in the study of polymer morphology.

\section{Acknowledgements}

The authors wish to thank the Perkin Elmer Co, Norwalk, Conn, USA, for their cooperation during the development of this special technique. The financial support of the National Aeronautics and Space Administration is gratefully acknowledged.

\section{References}

1. P. H. GEIL, J. Polymer Sci. C13 (1966) 149.

2. w. o. statton, "New Methods of Polymer Characterisation", Vol. 6, edited by B. Ke (Interscience, New York, 1964), pp. 231-78.

3. C. REINHOLD, E. W. FISCHER, and A. PETERLIN, J. Appl. Phys, 35 (1964) 71.

4. G. S. Y. YEH, Ph.D. thesis, Case Institute of Technology, Cleveland (November 1966).

5. H. VON MAHL and W. WEITSCH, $Z$. Naturforsch. A15 (1960) 1051.

6. G. A. BASSETt and A. KeILER, Phil. Mag. 9 (1964) 817.

7. P. H. GEIL, J. Polymer Sci. A2 (1964) 3825.

8. E. W. Fischer and G. schmid T, Angew. Chem. 74 (1962) 551.

9. P. H. GEIL, "Polymer Single Crystals" (Interscience, New York, 1963).

10. R. hosemann, J. Appl. Phys, 34 (1963) 25.

11. K. KOBAYASHI and M. KUROKAWA, Nature 196 (1962) 196.

12. K. L. Yudowitch, J. Appl. Phys. 22 (1951) 214.

13. H. кIно, (Dreyfus Lab, Durham, NC, USA), private communication. 
14. M. Woods and J. DoDge, (Case Institute of Technology, Cleveland, Ohio, USA), private communication.

15. P. E. DOHERTY and R. S. DAvis, J. Appl. Phys. 34 (1963) 619.

16. A. GUinier and G. Fournet, "Small Angle Scattering of X-rays" (Wiley, New York, 1955).
17. c. E. HALL, "Introduction to Electron Microscopy" (McGraw-Hill, New York, 1966).

18. H. C. VAN DE HULST, "Light Scattering by Small Particles" (Wiley, New York, 1957).

19. A. PETERLIN, P. INGRAM, and H. KIHO, Makromol. Chem. 86 (1965) 294. 